Infusionsther Transfusionsmed 1993;20(suppl 3):69-71

\title{
Autorenverzeichnis / Author Index
}

Arnold, R. F08-6, F09-6, Fll-

6, FP11-P1 Auchter, M. FP02-P6 Aulmann, M. FP02-P1, FP02-

P9 Afanasiev, B. SP09-P3

Balazs, E. S15-3

Bald, R. F06-5, FP13-P3

Barz, D. FP02-P20, FP05-P6,

FP13-P11 Bast, R. F07-4 Bauhaus,M. DP02-P12 Beaufort, F.FP12-P1 Beck, K.H. FP04-P4, F13-6,

F13-7, FP13-P5, FP13-P7 Bein, G. F10-1 Bellac, M. FP07-P2 Benn, H.P. F10-5, Fll-7, F132, FP13-P12, SP16-P1 Berlet, T. F13-1 Bewarder, S. SP06-P7 Beyer, J. D03-1, F09-5, FP10P5, SP03-P3, S14-2 Bindl, L. SP06-P1 Blanchard, D. FP04-P1 Blasczyk, R. F09-3, Fll-5 Bläsl, U. FP13-P2 Blauhut, B. D03-6, SP03-P4 Bleckmann, U. FP13-P5 Blumenberg, D. SP06-P3 Böcher, M. FP03-P9 Böck, M. D04-4, F06-4, F06-7,

F13-3, FP13-P1 Böhm, B.O. F10-6 Böhringer, M. D05-6, FP05-P3 Borberg, H. FP07-P2 Born, M. D04-8 Brandt, S. FP09-P2 Brauer, P. FP07-P1, F12-4 Bremer, K. F07-3 Brüster, H.T. FP10P7, FP10-

P8 Buchwald, S. F10-3 Budde, U. F13-8 Bunjes, D. F08-6, FP11-P1 Buroh, C. S15-2 Bux, J. D05-7, FP05-P2, SP06-

\section{$\mathrm{Pl}$}

Caspari, G. SP03-P3, S14-2 Cassens, U. F10-5, Fll-7,

F13-2, FP13-P9 Chen, D. F10-2 Chen, M. F10-6 Claus, R. F10-3

Dahr, W. D04-1, FP04-P1 Darda, C. SP16-P1 Decker, S. F07-4 Dengler, T. SP03-P2 Diekamp, U. FP02-P5, SP08-

Pl, S14-3, SP14-P6, SP14-P7 Dittmann, J. D02-2, F13-4 Dittmer, R. F10-5, Fl 1-7, F13-

2, SP16-P1 Dominka, T. FP02-P4, F10-5,

FP13-P9 Dopfer, R. FP13-P10 Dresow, B. F13-2 Drewke, E. F13-8 Drobnik, W. F07-2 Dupke, R. F10-1

Eckstein, R. DP02-P13, DP02-P14, D03-5, F08-4, F09-5, FP10-P3, FP10-P4, FP10-P5, FP10P6, F12-5, FP13-P8, SP03-P6, SP06-P8

Ehninger, G. Fll-6

Eichler, H. D04-5, FP04-P4, FP04-P6, SP14-P3

Eiermann, T./Th./T.H. F06-1, Fll-3, Fll-6

Enders, G. FP04-P11

Endres, W. FP04-P3, F10-2

Engelhardt, R. SP16-P1

Ennen, J. D03-2

Ernst, M. FP04-P1

Falk, U. F10-3

Feid,M. Fll-2 
Feller, K. D05-4

Feyerabend, K. SP15-P2

Fiebelkorn, A. F06-3

Fiedler, H. D03-2

Fischer, J. FP05-P7, F06-5,

F09-2, F12-2 Fischer, K. F12-7 Fischer, M. Fll-3 Fischer, R. F13-2, FP13-P12,

SP16-P1 Fölsch, B. SP03-P4 Frank, R. FP10-P1, Fll-2 Frank, S. FP02-P6 Fregatova, L. SP09-P3

Fricke, L. F10-1 Friedrich, W. F09-6 Fritze, H. D04-8 Fürst, G. SP03-P2

Gabbe, E.E. SP16-P1 Gabriel, C. D03-6, SP03-P4, S15-5

Ganschow, I. F13-2, FP13-P9,

FP13-P12 Gänshirt, K.H. S15-6 Garritsen, H.S.P. F12-4 Gawaz, M. F13-3 Gebhardt, B. FP02P20, SP03-

P8 Genter, P. SP03-P5 Gerlich, W.H. F13-1, SP03-P3,

SI 4-2 Gesemann, M. SP03-P5 Gielen, W. FP04-P1 Giers, G. F06-5, F09-2, F12-2,

F13-5, FP13-P3 Giesing, M. D03-2 Gilli, R. FP12-P1 Giptner, A. D05-6, FP05-P5 Gläser, R.

F10-1 Gliesche, Th. F07-4 Gohr, M. SP15-P1 Goldmann, S.F. Fll-3, Fll-6 Görg, S. F06-3

Gossrau, E. FP02-P2, FP13-

Pll Greher, I. D04-3 Greinacher, A. SP06-P5,

SP06-P6 Grosse-Wilde, H. Fll-6 Granenberg, R. F13-1 Grüntjes-Prinsen, G. F07-3 Gültekin, H. FP03-P10

Haase, D.F10-l, S15-3 Häberle, J. DP02-P11, FP02-

P18 Hack, H. FP04-P12 Haferlach, T. SP06-P9 Hammad-Zulfoghari, D. F07-3 Hanfland, P. FP05-P7, F06-5,

F09-2, F12-2, F13-5, FP13-

P3 Hansen, E. SI4-6 Hansmann, E. F07-3 Hansmann, M. F06-5 Hattstein, E. F06-2 Hausmann,

D. F10-3 Hausmann, S. F10-3 Haustein, B. FP04-P9 Hauswirth,W. FP13-P4 Heim, M.U. D04-4, F06-4,

F06-7, F13-3, FP13-P1 Heimpel, H. F08-6, F09-6,

FP11-P1 Heinze, B. F08-6 Hertenstein, B. F08-6, FP11-

Pl Heuberger, M. SP06-P2

Heuft, H.G. D03-5, FP04-P10,

F09-3, FP13-P8, SP06-P8 Heyll, A. F06-2 Hiller, J. D03-3, D05-5, FP02-

P4, FP13-P9 Hillringhaus, I. FP02-P5 Hinderer, W. SP03-P1 Hintz, G. FP13-P8 Hirsch, M.

SP08-P6 Hoch, J. F06-5, F09-2, FP13-

P3, SP06-P1 Holzberger, G. FP10-P1, Fll-

2, SP06-P2 Horn, J. SP03-P1 Hörnschemeyer, D. D03-1 Horstmann, E. FP02-P7 Hosoda, M.

FP02-P3 Huhn, D. FP04-P10, F09-3,

FP10-P4, FP10-P5, FP10-P6,

Fll-5, SP06-P8 Huss, B. D05-4, FP13-P5,

SP06-P7, SP14-P1

Ivens, B.FP13-P9

Jakobs, K. SP14-P2 Jiménez-Klingberg, C. FP07-

P2 Jung, F. FP13-P2, SP14-P2 Jürgens, H. FP07-P1

Kadar, J. D02-5, F08-3

Kalb, R. D05-2, FP05-P2, 
FP05-P3, F10-4 Kampe, D. F12-5 Kaspersen, K. D03-7 Kemper, J. F07-3 Kempter, B. FP13-P1 Kiefel, V. D05-2, D05-4, D05-

6, FP05-P1, FP05-P3, FP05-

P4, FP05-P5, F06-5 Kiesewetter, H. FP13-P2,

SP14-P2 Kirchner, H. DP02-P12, D03-

4, D03-8, FP02-P17, F06-3,

F10-1, SP03-P7, S15-3 Kirschfink, M. D04-9 Kleesiek, K. D03-1 Klingberg, J. FP07-P2 Klinge,

U. D04-5 Klinger, M. DP02-P12 Klouche, M. F06-3 Kluge, A. FP13-P10 Klüter, H. DP02-P12,

D03-4,

D03-8, FP02-P17, F06-3,

SP03-P7

70

Abstracts des 26. DGTI-Kongresses, Rostock, 16.-18. September 1993

Knoblauch, K.-D. D04-9 Knobloch, U. FP04-P8 Knoedler, B. D03-3, SP16-P1 Knop, D. DP02P16 Kober, B. D05-7 Koepsell, E. SP06-P2 Koerner, K. F09-6 Köhler, H.F10-3 Köhler, M. D022, D03-9,

FP12-P2, F13-4 Kokelj, M. FP02-P10 Kolb,H. Fll-6 Kollig, J. FP13-P6, SP14-P4 König, A.L. D04-6, FP04-P5,

S15-1 Köppler, H. SP06-P7 Koscielny, J. SP14-P2 Kostner, G.M. FP02-P10 Kovacs, E. F07-2

Krandick, E. FP02-P2 Krause, K.P. FP09-P2, SP08-

P2 Kreft, H. FP04-P12 Kretschmer, V. DP02-P11,

D04-5, D05-4, FP02-P18,

FP04-P4, FP04-P6,

F08-2, F13-6, F13-7, FP13-

P5, FP13-P7, SP06-P7,

SP08-P4, S14-1,

SP14-P1, SP14-P3 Kreutzig, B. S15-4 Kroll, H. D05-6, FP05-P1,

FP05-P3, FP05-P4, FP05-P5,

F06-5 Krüger, J. D03-7, F13-1,

SP06-P10 Kruse, A. D03-4 Krüssel, H. DP02-P8 Kubanek, B. D04-3, FP04-P2,

F08-6, F09-6, FP11-P1 Kucera, W. S14-5 Kühnl, P. D03-3, D05-5,

FP02-P4, F10-5, F10-6, Fll-

7, F13-2, FP13-P9,

FP13-P12, SP16-P1 Kunde, C. FP13-P9 Kunhardt, O. F12-5 Kuntz, B.M.E. F06-2, FP10-

P7, FP10-P8

Langner, A. FP12-P1

Lanzer, G. FP02-P10, FP10-

P2, FP12-P1, SP03-P4 Lattermann, A. Fl 1-4 Ledermann, A.E. F08-3 Leeping, M. D04-4, F06-7

Lehmann, P. FP04-P8 Leibundgut, K. F08-1 Lenhard, V. FP04-P8 Lenz, K. FP10-P5 Leo, A. FP04-P11

Lerche, D. S14-5 Lichy, S. FP04-P3 Liebenhoff, U. SP06-P5 Lindner, M. FP02-P1, FP02-P9

Löliger, C.-C. D03-3, D05-5,

Fll-7 Loth, F. FP02-P6 Lubitz, B. D05-5, FP02-P4 Lüdemann, K. F10-5 Ludwig, M. SP08-P8

Luz, B. S15-1 Lynen, R. FP12-P2

Maccari, B. F09-6, FP11-P1 
Mallmann, R. F09-2

Manouvriez, P. FP03-P11

Marzik, S. FP03-P11

Maschek, W. D03-6

Matthes, G. D02-1, D02-4, D02-5, FP02-P3, FP02-P6, FP02-P19, F08-5, F09-4, FP09-P1, FP09P2, SP08-P2, SP08-P3, S14-5, SP14-P5, SP14-P8, SP14-P10 SP14-Pll

Mavrina, L. FP02-P6

Mayer, G. D05-3

Melnikowa, W.N. FP02-P7

Mempel, W. D04-4, F06-4, F06-7, F13-3, FP13-P1

Meyer, S.A. Fl0-2

Michel, G.FP03-P 10

Mikolasch, A. D05-5

Minnert, G. FP03-P10

Mitschulat, H. F09-5

Möckl, M. SP06-P6

Mohr, P. FP13-P5

Moog, R. F08-3, SP08-P5

Morath, A. FP02-P9

Mortelmans, Y. F13-6, F13-7, FP13-P7, SP14-P1

Mross, E. D04-4

Mrowietz, C. FP13-P2

Mueller-Eckhardt, C. D05-2, D05-6, D05-7, FP05-P1, FP05-P2, FP05-P3, FP05-P4, FP05-P5, F06-5, SP06-P1, SP06-P5, SP06-P6

Mueller-Eckhardt, G. Fll-4

Muggenthaler, H. F06-4

Müller, N. D02-5, DP02-P8, DP02-P15, DP02-P17, FP02-P7, F08-2, F08-3, SP08-P4, SP08-P5, SP08-P7

Müller, W. SI5-2

Müller-Berghaus, G. FP13-P6, SP14-P4

Müller-Steinhardt, M. D03-8, FP02-P17

Muntean, W. FP05-P5

Nebel-Schickel, H. SP03-P1 Neidhardt, B. F08-4 Neppert, J. SP06-P9 Netz, M. FP02-P6, SP15P1 Neuhaus, R. FP12-P2 Neumann, H.-J. F08-2, SP08-

P4 Nielsen, P. SP16-P1 Niemann, K. D03-8 Northoff, H. D05-3 Novotny, J. F08-6 Nussbaumer, W. F06-6, SP08-

P6 Nydegger, U. F08-1

Oehler, M. FP13-P4 Offner R. SP08-P9 Ostkamp-Ostermann, P. FP07-Pl

Pachmann, U. SP08-P9 Pätzold, U. FP10-P2 Pawlow, I. FP02-P19, F08-5,

F09-4, FP09-P1, FP09-P2,

SP08-P2, SP08-P3, S14-5 Peters, J. SP06-P4 Petersen, N. D03-l, F07-3 Petrow, R. SP14-P8, SP14-P9,

SP14-P10 Pfeiffer-Wolf, I. FP13-P10 Pindur, G. FP13-P2, SP14-P2 Platanova, G. SP09-P3

Plattner, R. SP08-P6 Posch-Vuckovic, U. FP02-P10 Poschmann, A. F12-7 Presek, P. SP06-P5

Prochnow-Calcia, H. FP11-P1 Pruß, A. SP14-P5, SP14-P8,

SP14-P9, SP14-P10 Putzo, A. DP02-P13, DP02- 
P14

Quenzel, M.E. F12-2

Rath, B. FP07-P1

Rath, E. D02-4, FP02-P3, F09-4, FP09-P1, FP09-P2, SP08-P3

Rau, G. S14-4

Reckhaus, M.-L. FP13-P9

Reiser, H. F06-7

Reiser, M. F08-3

Richter, E. D02-1, D02-4, D02-5, FP02-P3, FP02-P19, F08-5, F09-4, FP09-P1, FP09-P2, SP08P2, SP08-P3, S14-5, SP14-P5, SP14-P10

Riess, H. DP02-P13, DP02-P14, FP13-P8

Riewald, M. DP02-P13, DP02-

P14 Riggert, J. D02-2, D03-9,

FP12-P2, F13-4 Roelcke, D. D04-9, FP04-P11,

FP04-P12, FP13-P10 Rood, J.J.vanFl 1-1 Roos, D. S15-2 Rossi, U.D01-1 Rothe, G. F07-2

Roushdy, J. F10-4 Ruf, K. FP02-P9 Ruth, N. SP08-P6 Rüther, G. FP13-P4

Saavedra, C. SP03-P1, SP06-

P2 Sachs, E. SP16-P2 Sachs, V. SP16-P2 Sänger, W. SP08-P9 Santoso, S. D05-2, D05-6, FP05-P1, FP05-P2, FP05-P3,

FP05-P5, F10-4 Sawodny, B. F09-6, FP11-P1 Schabel, A. D04-6, FP04-P5,

S15-1 Scharf, D. FP03-P11 Scharf, R.E. F06-5, F09-2,

F12-2, F12-3, F13-5, FP13-

P3 Scheiermann, N. SP03-P5 Scheld, S. FP05-P4 Schermuly, L. F08-2, SP08-P4 Schiebel, M.R. D04-6 Schleinzer,W. S14-4 Schleuning, M. FP13-P1 Schlosser, S. S14-6 Schlüter, C. D05-6, FP05-P5 Schmid, E. F06-6, SP08-P6 Schmidt, C. SP06-P9 Schmidt, R. F07-4 Schmitt, H. SP03P3, S14-2 Schmitz, G. FP04-P1, F07-2 Schmutzler, R. SP03-P1 Schnaidt, M. D05-3, FP05-P7

Schneider, C. FP07-P1 Schneider, W. F06-2 Schneidewind, J. F07-4 Schönitzer, D. F06-6, SP08-P6 Schoppet,M. Fll-4 Schöttler, B. FP10-P8 Schottstedt, V. D03-2 Schramek, G. SP08-P8 Schreiner, T. F08-6, F09-6,

FP11-P1 Schubert, C. F10-4 Schultze, W. F09-4, FP09-P1,

FP09-P2 Schulz, R. SP06-P10 Schulzki, T. DP02-P11, FP02-

P18, SP14-P1

Abstracts des 26. DGTI-Kongresses, Rostock, 16,-18. September 1993

71

Schürger, G. SP06-P3 Schuster, M. FP02-P19 Schwaller, J. F08-1 Schwarz, D. SP16-P1

Schwarz, K. Fll-7 Schwella, N. D03-5, FP04-

P10, F09-3, Fll-5, F12-5,

FP13-P8, SP06-P8 Schwerdtfeger, R. F09-5 Sehland, D. F07-4 Seidel, K. D03-1 Seidl,C. FP10Pl, Fll-2 Seifertova, M. FP04-P9 Seifried, E. FP10-P1, Fll-2,

SP06-P2 Sekiguchi, S. FP02-P3 Selle, B.F07-1 Semmler, C. FP10-P1 Serke, S. F12-5

Sibrowski, W. FP07-P1, F12-4 Simpson, B. FP03-P10 Simson, G. D02-2, D03-9,

FP12-P2, F13-4 Singbartl, G. S14-4 Sohn, M. D05-7 Söhngen, D. F06-2 Sonneborn, H.-H. D042, 
FP04-P1.SP03-P1 Späth, P. FP07-P2 Sputtek, A. S14-4 Stangel, W. FP04-P3, F10-2 Stefanic, M. F08-6, FP11-P1 Steigerwald, A. SP06-P3 Stein, E.-L. FP05-P2 Steinhagen-Thiessen, E. FP10-

P6

Storch, H. F12-1

Strobel, E. D04-7, FP04-P7, SP06-P4 Strohscheer, I. FP10-P5 Struve-Holke, S. SP06-P9 Sturmfels, L. FP04-P8 Stuth, C. FP04-P5 Sugg, U. D04-6, FP04-P5,

S15-1 Sutherland, R.FP03-P 10 Suttorp, M. SP06-P9

Taborski, U. FP13-P6, SP14-

P4 Taeger, K. S14-6 Takahashi, T.A. FP02-P3 Thierbach, V. SP08-P8 Tietze, M. SP03-P7

Tofoté, U. D02-1, D02-4,

FP02-P3, F08-5, SP08-P3,

S14-5 Troch, M. SP03-P7 Truschnig-Wilders, M. FP12-

Pl Tuma, W. D03-2

Ullrich, H. F07-2 Ullrich, R. FP02-P1, FP02-P9 Ulrich, S. FP10-P2 Unkelbach, K. FP05-P1

Uthemann, H. FP04-P8

Vehmeyer, K. D03-9 Vockel, A. FP03-P9

Volkova, O. SP09-P3 Vornhagen, R. SP03-P1 Vornwald, A. FP02-P2

Wagner, D. FP02-P20 Wagner, F. D04-3, FP04-P2,

F12-6 Wagner, H.J. D03-4 Wagner, R.SP14-P11 Walka, M. FP05-P3 Walker, W.H. FP02-P6, SP15-

Pl Walther-Wenke, G. FP02-P7,

FP13-P4 Weber, S. SP06-P7 Wegener, S. F10-3 Weippert-Kretschmer, M. S14-

1 Weisbach, V. DP02-P13,

DP02-P14, D03-5, F08-4,

F09-5, FP10-P5, FP10-P6,

F12-5, SP03-P6 Weise, W. FP03-P11 Weißhaar, H.-D. S15-4 Weißwange, T. F08-2, F08-3, SP08-P4 Wenzel, E. FP13-P2, SP14-P2 Wernet, D. D05-3 Wester, S. Fl 2-7 Wichmann, M.G. SP06-P9 Wiebecke, D. SP06-P3 Wieding, J.U. D02-2, D03-9,

F13-4 Wiesneth, M. F08-6, F09-1,

F09-6, FP11-P1

Wilhelm, D. F06-3 Witt-Meisel, T. FP13-P11 Wittmann, G. FP04-P10,

FP10-P3, FP10-P4, FP10-P6,

FP13-P8, SP03-P6, SP06-P8 Wolf, G. FP09-P1 Wolff, C.D03-1 Wolfsdorff, B. F07-4 Wölpl, A.

D04-3, FP04-P2,

Fll-3 Worschech, W. SP08-P9 Wüllenweber, J. D04-7, FP04-

P7, SP06-P4

Zander, A. Fll-7

Zang, Y. SP06-P9

Zeiler, T. DP02-P13, DP02-P14, FP04-P10, F08-4, F09-3, F09-5, FP10-P3, FP10-P4, F12-5, FP13-P8, SP03-P6, SP06-P8

Zilow, E.P. F07-1

Zilow, G. F07-1

Zimmermann, B. FP02-P5 
Zimmermann, R. DP02-P13, DP02-P14, D03-5, F08-4, F09-3, F09-5, FP10-P3, FP10-P4, FP10P6, Fll-5, F12-5, FP13-P8, SP03-P6

Zingsem, J. DP02-P13, DP02-P14, F08-4, F09-5, FP10-P3, FP10-P4, FP10-P5, FP10-P6, F12-5, SP03-P6

Zumpe, P. SP06-P2 